\title{
Significance of elevation of the right dome of the diaphragm
}

\author{
S. RAMACHANDRAN * \\ M.D., M.R.C.P., M.R.C.P.E.
}

\author{
D. L. N. JAYAWARDENA \\ M.B.B.S., D.M.R.D.(Lond)
}

\author{
M. B. WARAKAUlle \\ M.B.B.S., T.D.D., D.M.R.D.
}

* The Colombo North General Hospital, Ragama, and the Colombo South Hospital, Kalubowila, Sri-Lanka

\begin{abstract}
Summary
Elevation of the right dome of the diaphragm occurs in a small proportion of healthy young adults, and in a proportion of patients with cardiac disease, cirrhosis of the liver and gastroenteritis. Hence this radiological feature, although common in hepatic amoebiasis, should be considered in concurrence with the other clinical features in the diagnosis of hepatic disorders. The significance of an elevation in the right hemidiaphragm in diseases primarily non-hepatic in origin, although of no diagnostic importance, is of clinical interest and is discussed.
\end{abstract}

\section{Introduction}

Elevation of the right dome of the diaphragm is a common radiological abnormality in the syndrome of hepatic amoebiasis (Ramachandran, Jayawardena and Perumal, 1971). Consequently, it has been included as a criterion in the clinical diagnosis of the syndrome (Lamont and Pooler, 1958). It therefore becomes necessary to establish the frequency of this radiological sign in healthy individuals, and in patients with other medical disorders in geographical locations where hepatic amoebiasis is prevalent.

\section{Materials and methods}

Postero-anterior and right lateral chest $\mathrm{X}$-rays were taken in (i) 111 healthy adult males (aged 20 to 38 years, mean 24 years); (ii) 268 patients, with various medical conditions, admitted to the Medical Units of the Base Hospital, Chilaw, and the Colombo North General Hospital, Ragama, Sri-Lanka. These medical conditions included:

(a) Pulmonary diseases: acute and chronic bronchitis; tropical eosinophilia; lung consolidation and abscess; tuberculosis (eighty-five patients-fifty-six male, twenty-nine female, aged 13 to 83 years, mean 48 years).

(b) Cardiac disease: ischaemic heart disease;

Correspondence: Dr S. Ramachandran, Colombo North General Hospital, Ragama, Sri-Lanka. hypertension and heart disease secondary to anae- $\frac{8}{3}$ mia (twenty-nine patients-sixteen male, thirteen iv female, aged 15 to 80 years, mean 49 years).

(c) Fevers (eighteen patients-thirteen male, five female, aged 12 to 60 years, mean 34 years).

(d) Gastroenteritis: amoebic and bacillary dysen. tery (nineteen patients-sixteen male, three female, 은 aged 14 to 35 years, mean 29 years).

(e) Cirrhosis of the liver (fifty patients-forty-six $\vec{z}$ male, four female, aged 32 to 80 years, mean 47 years).

(f) Viral hepatitis of short and long incubatiog $\overrightarrow{0}$ (forty-five patients-twenty-seven male, eightee oे female, aged 12 to 60 years, mean 24 years).

(g) Other disorders, e.g. rheumatoid arthritiș cervical spondylosis, myalgic chest pain (twenty-five patients-twelve male, ten female, aged 16 to 75 years, mean 42 years).

Elevation of the right dome of the diaphragm was diagnosed when it was $3.5 \mathrm{~cm}(1.4 \mathrm{in})$ or higher than the left. This value for the difference in the domes, although higher than the figure usually used $(2.5 \mathrm{~cm}$ or 1.0 in), would prevent over-reading of the X-rays (Ramachandran, Warakaulle and Vinayagamurthy, 1975).

\section{Results}

(i) Healthy subjects. Elevation of the right dome of the diaphragm was present in two of the 111 $(1.9 \%)$ (Ramachandran et al., 1975).

(ii) Patients. The incidence of elevation of the right $\frac{D}{0}$ dome in some of the commoner medical disorders is listed in Table 1. There was a striking incidence in $\tilde{N}$ patients with cardio-vascular disease $(34.5 \%)$, N cirrhosis of the liver $(34 \%)$ and gastroenteritis 0 $(10.5 \%)$. However, the incidence in patients with $\omega$ viral hepatitis was low. The overall frequency of this radiological sign in the group of 268 cases was $14.5 \%$.

\section{Discussion}

Radiological surveillance establishes the fact that 
TABLE 1. The incidence of an elevation of the right dome of the diaphragm in patients with some common medical disorders

\begin{tabular}{lccc}
\hline Medical disease & $\begin{array}{c}\text { Number of } \\
\text { cases } \\
\text { X-rayed }\end{array}$ & $\begin{array}{c}\text { Elevation of } \\
\text { the right } \\
\text { dome }\end{array}$ & $\%$ \\
\hline Pulmonary disease & 85 & 6 & $7 \cdot 1$ \\
Cardiac diseases & 29 & 10 & $34 \cdot 5$ \\
Fever & 18 & - & - \\
Gastroenteritis & 19 & 2 & $10 \cdot 5$ \\
Cirrhosis of liver & 50 & 17 & $34 \cdot 0$ \\
Viral hepatitis & 45 & 2 & $4 \cdot 4$ \\
Miscellaneous & 22 & 2 & $9 \cdot 1$ \\
& 268 & 39 & $14 \cdot 5$ \\
\hline
\end{tabular}

elevation of the right dome of the diaphragm occurs in a small proportion of healthy young adults, and in a high proportion of patients with cardiac disease, cirrhosis of the liver and gastroenteritis. It follows that this radiological sign is not peculiar to the syndrome of hepatic amoebiasis.

The occurrence of an elevation of the right hemidiaphragm in otherwise healthy young adults is relevant to the certifying of medical fitness for life insurance. The aetiological factors producing elevation could be either abnormal posture and spinal deformities, congenital or acquired weakness of the diaphragmatic musculature with or without visceral herniation, or an upward enlargement of the liver (Simon, 1962). Whereas the first factor would be obvious in the chest X-rays, screening of the chest and tests for diaphragmatic movement would be necessary for detection of the latter causes.

Evaluation of the significance of this radiological sign in medical disorders is best considered, firstly, with reference to primary diseases of the liver and, secondly, in relation to diseases where hepatic involvement is of secondary importance. There is no doubt as to the value of this abnormal sign in the diagnosis of hepatic amoebiasis, whether pus is demonstrated or not. The rarity of this feature in patients with viral hepatitis and leptospirosis further indicates its clinical value. Nevertheless, its occurrence in hepatic disorders such as cirrhosis and malignancies of the liver (Rowland, 1963) shows that this radiological sign should be considered in relation to other features in the diagnosis of liver diseases, including hepatic amoebiasis.

The significance of an elevated right hemidiaphragm in diseases primarily non-hepatic in origin, although of no diagnostic importance, is of clinical interest. In patients with cardiac disease it would indicate the presence of congestive cardiac failure, while in those with colonic ulceration it suggests secondary hepatic involvement. Hepatic involvement has been recognized in ulcerative colitis and regional enteritis (Dordal, Glagov and Kirsner, 1967), and in intestinal amoebiasis (Ramachandran, Sivalingam and Perumal, 1973; Ramachandran et al., 1973). Further to this study, this radiological feature has been observed in neoplastic diseases such as chronic myeloid leukaemia. Phrenic nerve involvement should be excluded in cases of elevation of the right dome of the diaphragm in pulmonary disease.

\section{References}

Dordal, E., Glagov, S. \& Kirsner, J.B. (1967) Hepatic lesions in chronic inflammatory bowel disease: Clinical correlations with liver biopsy diagnosis in 103 patients. Gastroenterology, 52, 239.

LAmont, N. McE. \& Pooler, N.R. (1958) Hepatic amoebiasis. Quarterly Journal of Medicine, 27, 390.

Ramachandran, S., Jayawardena, D.L.N. \& Perumal, J.R.A. (1971) Radiological changes in hepatic amoebiasis. Postgraduate Medical Journal, 47, 615.

Ramachandran, S., Sivalingam, S. \& Perumal, J.R.A. (1973) Concepts in hepatic amoebiasis. Journal of Tropical Medicine and Hygiene, 76, 39.

Ramachandran, S., de Saram, R., Rajapakse, C.N.A. \& SivalingaM, S. (1973) Hepatic manifestations during amoebic dysentery. Postgraduate Medical Journal, 49, 261.

Ramachandran, S., Warakaulle, M.B. \& VinayagaMURTHY, V. (1975) The diaphragm in chest radiology. Medical Journal of Australia, 1, 680.

RowLAND, H.A.K. (1963) Radiology of amoebic liver abscess. Journal of Tropical Medicine and Hygiene, 66, 113.

Simon, G. (1962) Principles of Chest X-ray Diagnosis. Butterworth \& Co. Ltd: London. 